\title{
Remote Synchronization Simulation of Onboard Crystal Oscillator for QZSS Using L1/L2/L5 Signals for Error Adjustment
}

\author{
Toshiaki Iwata, ${ }^{1}$ Michito Imae, ${ }^{1}$ Tomonari Suzuyama, ${ }^{1}$ Yuji Hashibe, ${ }^{2}$ Satoshi Fukushima, ${ }^{2}$ \\ Akira Iwasaki, ${ }^{3}$ Kenji Kokubu, ${ }^{3}$ Fabrizio Tappero, ${ }^{4}$ and Andrew G. Dempster ${ }^{4}$ \\ ${ }^{1}$ National Institute of Advanced Industrial Science and Technology (AIST), Tsukuba, Ibaraki 305-8568, Japan \\ ${ }^{2}$ Space Engineering Development Co. Ltd., Tokyo, Nakano-ku, Tokyo 164-0001, Japan \\ ${ }^{3}$ Department of aeronautics and astronautics, Faculty of Engineering, University of Tokyo, Tokyo 113-8656, Japan \\ ${ }^{4}$ School of Surveying and Spatial Information Systems Faculty of Engineering, University of New South Wales, Sydney, \\ NSW 2052, Australia
}

Correspondence should be addressed to Toshiaki Iwata, totty.iwata@aist.go.jp

Received 17 July 2007; Accepted 6 October 2007

Recommended by Demetrios Matsakis

A new error adjustment method for remote synchronization of the onboard crystal oscillator for the quasi-zenith satellite system (QZSS) using three different frequency positioning signals (L1/L2/L5) is proposed. The error adjustment method that uses L1/L2 positioning signals was demonstrated in the past. In both methods, the frequency-dependent part and the frequency-independent part were considered separately, and the total time information delay was estimated. By adopting L1/L2/L5, synchronization was improved by approximately $15 \%$ compared with that using L1/L2 and approximately $10 \%$ compared with that using L1/L5 and a synchronization error of less than 0.77 nanosecond was realized.

Copyright (C) 2008 Toshiaki Iwata et al. This is an open access article distributed under the Creative Commons Attribution License, which permits unrestricted use, distribution, and reproduction in any medium, provided the original work is properly cited.

\section{INTRODUCTION}

The Japanese Quasi-Zenith Satellite (QZS) System (QZSS) is a three-satellite navigation/positioning system conceived to improve the positioning performance (satellite availability and position accuracy) of the presently available global positioning system (GPS) in urban areas where high-rise buildings reduce the number of visible GPS satellites [1]. A new timekeeping method of the QZSS, named the remote synchronization system for an onboard crystal oscillator (RESSOX), has been planned by the National Institute of Advanced Industrial Science and Technology (AIST, Tasukuba, Japan) [2]. RESSOX is a remote-control method that permits synchronization between a ground station standard and QZS clocks. In its original concept, various delay models are used for the estimation of the delay of the RESSOX control signal that includes time information of QZSS time and is advanced with respect to QZSS-time to compensate the delay during transmission. This is considered to be the feed-forward control. Furthermore, pseudoranges of positioning signals ob- tained at the ground station, named the time management station (TMS), are used for error adjustment, where QZSSTime is a standard time of QZSS, like GPS time for GPS, and refers to UTC (NICT). This is considered to be the feedback control. RESSOX is realized by combining feed-forward and feedback control. The RESSOX control signal is transmitted with the $K u$-band from the TMS.

The proposed Japanese QZSS has the following properties regarding its timekeeping system (TKS): (1) it is possible to control the system over a 24-hour period as long as good visibility of QZS is obtained; (2) a high-stability crystal oscillator is superior to an atomic standard in terms of short-term frequency stability [3]; and (3) the QZSS employs a maximum of three satellites, which are not too many to monitor from the ground.

RESSOX reduces overall costs, satellite power consumption, and onboard weight and volume; and it has a longer lifetime than a system with onboard atomic clocks.

RESSOX ground experiments and computer simulations have been conducted since 2003. QZS broadcasts four 
positioning signals as availability enhancement signals: L1C/A, L1C, L2C, and L5 [4]. The tentative target of our research is synchronization to within 10 nanoseconds between the ground standard time and the QZS site at any time and frequency stability better than $1 \times 10^{-13}$ for 100,000 seconds. Initial experimental results using only L1/L2 positioning signals and experimental apparatus have been introduced previously [5-10]. We have developed a new feedback method that uses L1/L2/L5 positioning signals of the QZSS and proved that we could improve synchronization by $15 \%$ compared with the former L1/L2 method. Since experimental apparatuses for L5 are not available at the moment, only simulation results are presented in this paper.

Evaluations of the effects of the range error magnitude and the least-squares filter used at the ground site are also discussed.

\section{SIMULATION MODEL}

To investigate this new RESSOX method, a specific software simulator has been developed. The actual onboard crystal oscillator is MINI-OCXO manufactured by C-MAC MicroTechnology (Buckinghamshire, UK), and is modeled as follows:

$$
f=1.023 \times 10^{7}\left(1.0+3.2500048 \times 10^{-8}(V-5.352333)\right),
$$

where $f$ is the output frequency and $V$ is the applied voltage (when $V=5.352333 \mathrm{~V}, f=10.23 \mathrm{MHz}$ ).

To control MINI-OCXO using the difference between uplinked time information with RESSOX control signal and MINI-OCXO time, modified PI control of the control voltage was employed. The following formula that describes modified PI control was used:

$$
\begin{aligned}
v_{k}= & \text { offset }-\frac{k_{1}}{l+1} \sum_{i=k-l}^{k}\left(t_{\text {OCXO }}-t_{\text {RESSOX }}\right)_{i} \\
& -k_{2} \sum_{i=0}^{k-1} \int_{i}^{i+p}\left(t_{\text {OCXO }}-t_{\text {RESSOX }}\right) d t .
\end{aligned}
$$

Here, $v_{k}$ is the $k$ th output voltage, offset $=5.352333(\mathrm{~V}), k_{1}$ is a proportional gain set at $7.0 \times 10^{6}, k_{2}$ is an integral gain set at $3.0 \times 10^{4}, l$ is the number of past data used for proportional control and is set at $1, k$ is the data number from the beginning of the simulation, $p$ is the integral interval, which means an overlapping integral number, set at 2 , and $t_{\text {RESSOX }}$ is time information of the received RESSOX control signal.

Control repetition at the TMS is once every second, and that on the QZS is once every 1.5 seconds.

The simulation conditions are shown in Table 1. Typical Keplerian orbit elements of the QZS, shown in Table 1, were assumed. To calculate the orbit precisely, the EGM96 geopotential model with the spherical harmonic coefficient of degree and order 360, the gravity effects of the sun, the moon, and other planets taken from the Jet Propulsion Laboratory (JPL, NASA, Pasadena, Calif, USA) ephemeris DE405, the radiation pressure, and the solid tide effects were considered. To calculate ionospheric delay, data (COD10426.ION)

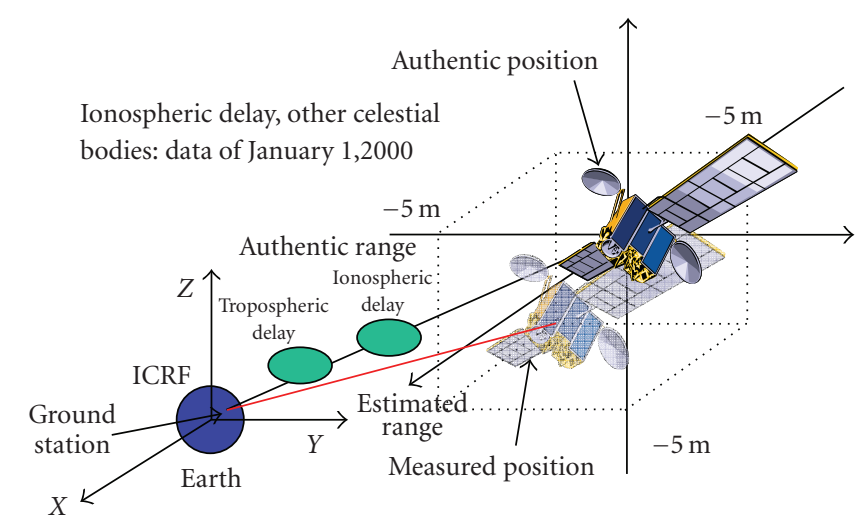

Figure 1: Differences in initial conditions between authentic delay and estimated one.

from the Center for Orbit Determination in Europe (CODE, University of Bern, Bern, Switzerland) was used. The simulation period was all day, January 1, 2000. This means that positions of the sun, the moon, and other planets and ionospheric data for that day were used. The meteorological conditions for tropospheric delay calculation were assumed to be constant at $15^{\circ} \mathrm{C}, 1013.25 \mathrm{hPa}$, and $70 \%$ relative humidity, and the Saastamoinen model was used. The position of the TMS was assumed to be in Okinawa $(26.5 \mathrm{~N}, 127.9 \mathrm{E}$, elevation $=0.0 \mathrm{~m})$. The calculations using these parameters correspond to the authentic range in Figure 1, and the "Orbit/Delay calculation (without error)" in Figure 2. These conditions can be expressed as $x=-22,881,059.583 \mathrm{~m}, y=-32,625,645.367 \mathrm{~m}, z=$ $19,898,922.824 \mathrm{~m}, v_{x}=2,207.153 \mathrm{~m} / \mathrm{s}, v_{y}=-839.448 \mathrm{~m} / \mathrm{s}$, and $v_{z}=1,693.581 \mathrm{~m} / \mathrm{s}$ as the initial conditions in the International Celestial Reference Frame (ICRF).

For the orbit information used at the TMS, an initial error of $-5 \mathrm{~m}$ for each axis of ICRF, that is, the initial conditions of $x=-22,881,064.583 \mathrm{~m}, y=-32,625,640.367 \mathrm{~m}$, and $z=19,898,917.824 \mathrm{~m}$ of the equation of motion $\left(v_{x}, v_{y}\right.$, and $v_{z}$ are the same as the authentic values) are assumed in order to create the time adjustment file for the transmitting time adjuster (TTA) and the database of L1/L2/L5 in Figure 2. The ionospheric and tropospheric delays were not considered. The differences in initial conditions between authentic delay and estimated delay are shown in Figure 1.

\section{CONTROL METHOD}

To realize RESSOX, L1, L2, and L5 pseudoranges were considered separately, and the delay of the frequency-dependent part (i.e., ionospheric delay) and that of the frequencyindependent part (i.e., clock error, range error, and tropospheric delay) were estimated. The following is the simulation sequence of this new method, as shown in Figure 2. In the simulation, some experimental apparatuses, such as the onboard crystal oscillator (MINI-OCXO), TTA, the time comparator, and the QZS signal receiver are modeled based on the ground experiments. 
TABLE 1: Simulation conditions.

\begin{tabular}{|c|c|c|c|}
\hline Items & Values & Items & Values \\
\hline \multirow{2}{*}{ Simulation period } & $\begin{array}{c}\text { 2000.1.1 } \\
\text { 00:00:00UTC- }\end{array}$ & Satellite cross section, $\mathrm{m}^{2}$ & 30.0 \\
\hline & $\begin{array}{c}\text { 2000.1.2 } \\
\text { 00:00:00UTC }\end{array}$ & CODE data of ionosphere & COD10426.ION \\
\hline Semimajor axis, $\mathrm{m}$ & 42164170.0 & \multirow{2}{*}{ Meteorological condition } & \multirow{2}{*}{$\begin{array}{l}15^{\circ} \mathrm{C}, 1013.25 \mathrm{hPa}, 70 \% \text { (relative } \\
\text { humidity) }\end{array}$} \\
\hline Eccentricity, $\mathrm{m}$ & 0.099 & & \\
\hline Inclination, deg & 45.0 & \multirow[b]{2}{*}{ Radiation pressure coefficient $(\mathrm{Cr})$} & \multirow[b]{2}{*}{$4.56 \times 10^{-6} \mathrm{~N} / \mathrm{m}^{2}($ McCarthy 1996), $\mathrm{Cr}=1.2$} \\
\hline $\begin{array}{l}\text { Right ascension of the } \\
\text { ascending node, deg }\end{array}$ & 205.0 & & \\
\hline Argument of perigee, deg & 270.0 & Position of ground station & $26.5 \mathrm{~N}, 127.9 \mathrm{E}$, Height $=0.0 \mathrm{~m}($ Okinawa $)$ \\
\hline Mean motion, deg & 120.0 & Solid Earth tide & Moon and Sun are considered, k2 = 0.3 (IAG 1999) \\
\hline Geopotential model & EGM96, $\mathrm{n}, \mathrm{m}=360$ & \multirow[t]{2}{*}{ Other celestial bodies } & $\begin{array}{l}\text { Moon, Sun, Mercury, Venus, Mars, } \\
\text { Jupiter, Saturn, Uranus, Neptune, }\end{array}$ \\
\hline Satellite mass, $\mathrm{kg}$ & 3000.0 & & Pluto(JPL-DE405) \\
\hline
\end{tabular}

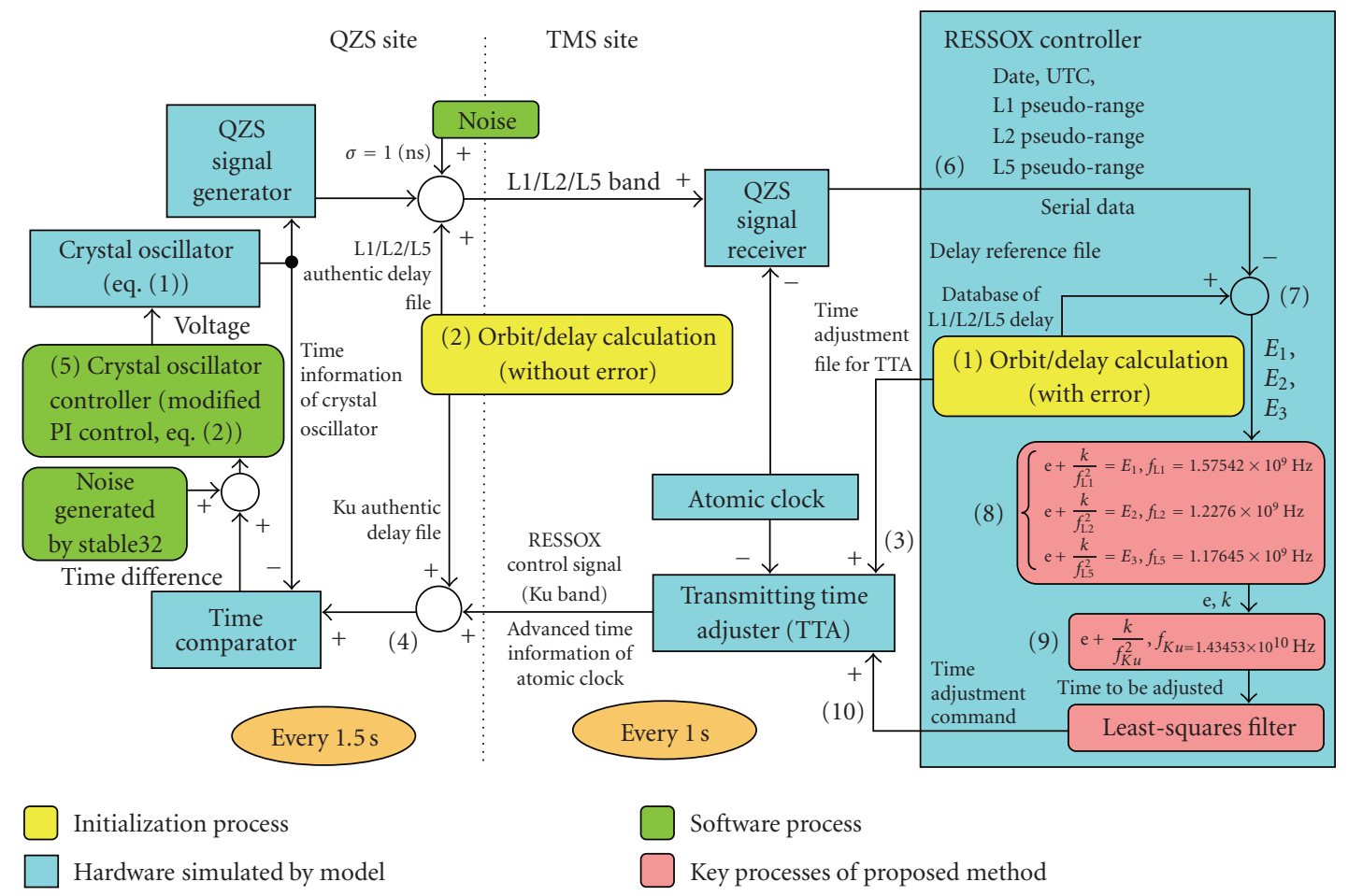

FIgURE 2: Simulation block diagram. Number in parenthesis indicates the step explained in the text. Our goal is synchronization between a ground atomic clock at TMS and an onboard crystal oscillator on QZS.

\section{Initialization (Steps 1 to 3)}

Step 1. Four estimated delays (L1-, L2-, L5-, and $\mathrm{Ku}$-bands) are prepared. These estimated delays include model errors such as those due to the orbit, ionosphere, or troposphere, and we assume that they are used at the TMS as the measurement results. The estimated delays of the L1-, L2-, and L5-bands make up the database of L1, L2, and L5 delays in the RESSOX controller to be used for comparison with the
L1-, L2-, and L5-band pseudoranges in Step 7. In contrast, the estimated delay of the $K u$-band is described in the time adjustment file for TTA, and is used as feed-forward control.

Step 2. Four authentic delays (L1-, L2-, L5-, and $K u$-bands) are prepared. These delays do not contain any errors. Three of these delays are contained in the L1, L2, and L5 authentic delay file, and the fourth one is contained in the $K u$ authentic delay file. 


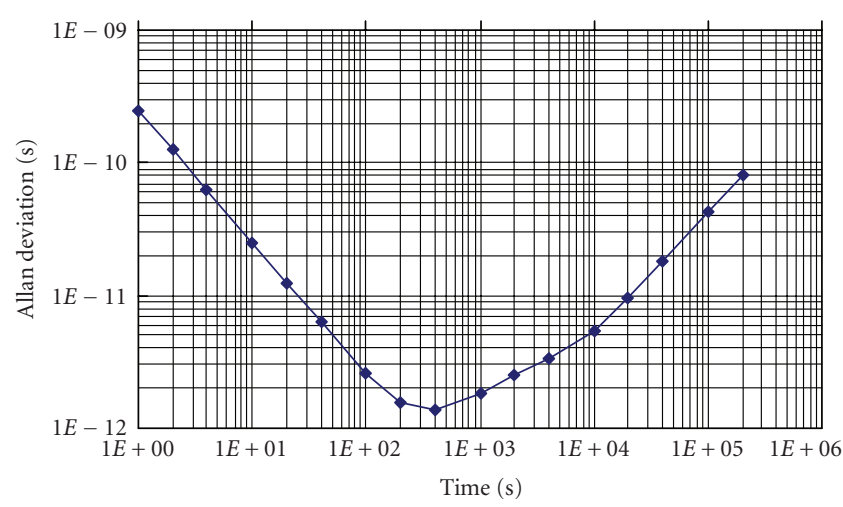

Figure 3: Assumed allan deviation of onboard system.

Step 3. The time adjustment file for TTA is fed into the TTA as feed-forward control. The timing for transmitting time information using the RESSOX control signal is adjusted to give the time comparator the correct time when the signal arrives at the QZS.

\section{Process routine (Steps 4-10)}

Step 4. The delay of the RESSOX control signal during transmission is realized by the $K u$ authentic delay file.

Step 5. The onboard crystal oscillator is controlled using the time difference between the RESSOX control signal and the time of the crystal oscillator itself. Some noise generated by the crystal oscillator and the time comparator is assumed in this step and is generated by Stable 32, a clock-simulation software [11]. We assume that the onboard crystal oscillator has a stability of $1.0 \times 10^{-12}$ from 1 to 100 seconds and $5.0 \times$ $10^{-11}$ for one day $(86400 \mathrm{~s})$, giving random walk frequency noise $=5.0 \times 10^{-14}$, flicker frequency noise $=6.5 \times 10^{-13}$, and frequency drift per second $=6 \times 10^{-16}$, and the time comparator has a stability of $2.5 \times 10^{-10}$ for 1 second and has only phase-white noise. The Allan deviation is shown in Figure 3.

Step 6. The pseudoranges of L1, L2, and L5 are calculated using the L1, L2, and L5 authentic delay file and the onboard crystal oscillator error. Noise that has 1 nanosecond standard deviation is added during transmission.

Step 7. The pseudoranges of L1, L2, and L5, obtained by the QZS signal receiver, are compared with the database of L1, L2, and L5 delay, and the differences between the pseudoranges and the database are designated as $E_{1}$ for L1 (frequency $\left.f_{L 1}=1.57542 \times 10^{9} \mathrm{~Hz}\right), E_{2}$ for $\mathrm{L} 2\left(f_{L 2}=1.2276 \times 10^{9} \mathrm{~Hz}\right)$, and $E_{3}$ for $\mathrm{L} 5\left(f_{L 5}=1.17645 \times 10^{9} \mathrm{~Hz}\right)$.

Step 8. Simultaneous equations (3), which include $E_{1}, E_{2}$, and $E_{3}$, delays due to the nonfrequency-dependent term $e$, and the coefficient of delay $k$ due to the frequency-dependent

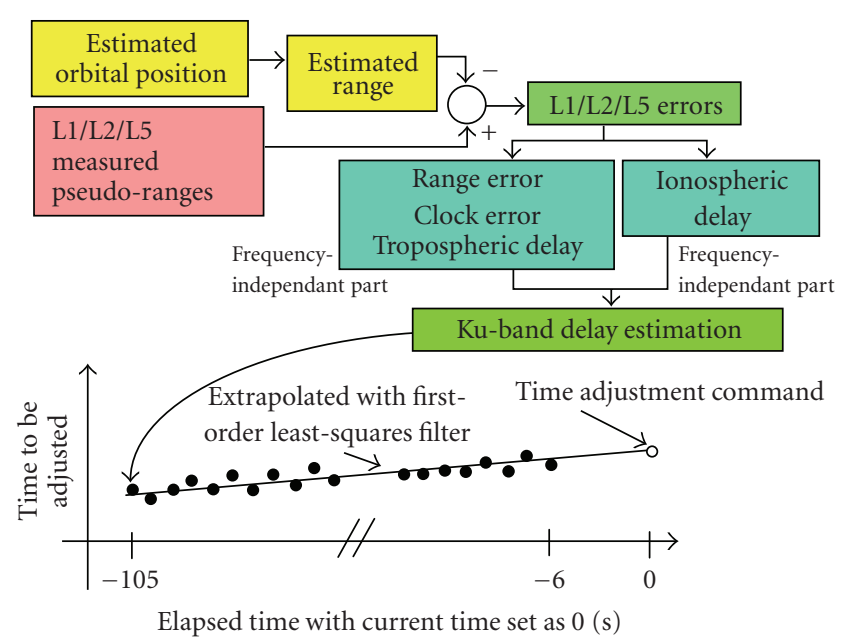

FIGURE 4: Default control method at TMS.

term (i.e., ionospheric delay) as unknowns, are solved:

$$
\begin{aligned}
& e+\frac{k}{f_{L 1}^{2}}=E_{1}, \quad f_{L 1}=1.57542 \times 10^{9}[\mathrm{~Hz}], \\
& e+\frac{k}{f_{L 2}^{2}}=E_{2}, \quad f_{L 2}=1.2276 \times 10^{9}[\mathrm{~Hz}], \\
& e+\frac{k}{f_{L 5}^{2}}=E_{3}, \quad f_{L 5}=1.17645 \times 10^{9}[\mathrm{~Hz}] .
\end{aligned}
$$

These equations are expressed using a matrix as follows:

$$
\left[\begin{array}{ll}
1 & 1 / f_{L 1}^{2} \\
1 & 1 / f_{L 2}^{2} \\
1 & 1 / f_{L 5}^{2}
\end{array}\right]\left[\begin{array}{l}
e \\
k
\end{array}\right]=\mathbf{A x}=\left[\begin{array}{l}
E_{1} \\
E_{2} \\
E_{3}
\end{array}\right]=\mathbf{E} .
$$

Since $\mathbf{A}$ is not a square matrix, to solve the equations, pseudoinverse $\mathbf{x}=\left(\mathbf{A}^{T} \mathbf{A}\right)^{-1} \mathbf{A}^{T} \mathbf{E}$ is used.

Step 9. Using the solutions of the simultaneous equations, we obtain the time to be adjusted with formula (5), of the RESSOX control signal using the $K u$-band $\left(f_{K u}=1.43453 \times\right.$ $10^{10} \mathrm{~Hz}$ ) for the TTA

$$
e+k / f_{K u}^{2}, \quad f_{K u}=1.43453 \times 10^{10}[\mathrm{~Hz}] .
$$

Step 10. As a result of combining the delay estimation file in Step 3 and the time to be adjusted for the TTA, the TTA is controlled. This process is considered to be the feedback control. We consider some filters in this step, as described later. Then we go back to Step 4. The calculation of the time to be adjusted is conducted every second. The default filter is constructed using 100 data values of the time to be adjusted (result of formula (5) using the difference between measured pseudoranges of L1/L2/L5 and estimated pseudoranges of L1/L2/L5 prepared as the database of L1/L2/L5 delay) from 6 to 105 seconds before every second. In our first consideration, the change of the time to be adjusted would depend on mainly the tropospheric delay in Figure 4. Since tropospheric delay depends on the elevation angle, the change of tropospheric delay can be approximated in the first order for such 


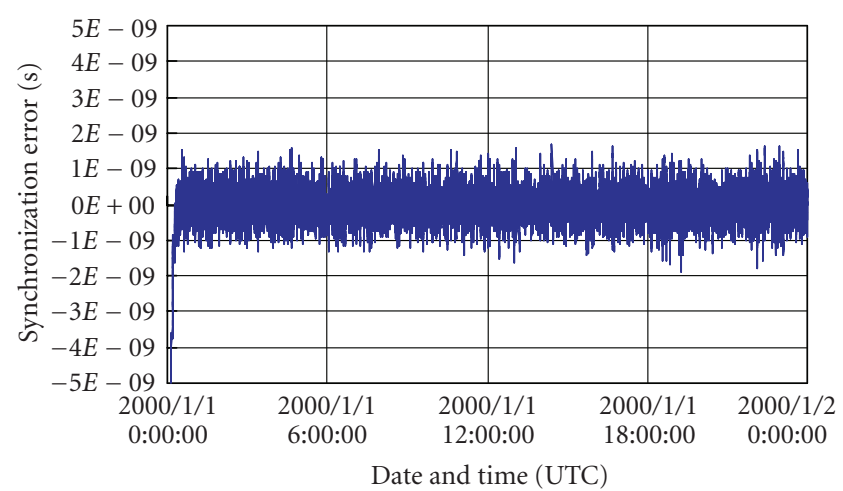

FIgURE 5: Synchronization result. The synchronization is within 1 ns.

a short period as 100 seconds. Therefore, 100 data values of time to be adjusted are used for the first-order least-squares filtering, and the time to be adjusted is extrapolated to the current time, as shown in Figure 4. To calculate and send the filtering result to the TTA as the time adjustment command, six seconds are assumed to be required.

Control is conducted every second on the ground and every 1.5 seconds on the QZS. These control frequencies will be actually adopted in the QZSS project.

In Figure 2, the three pink blocks indicate the key processes of this method.

\section{SIMULATION RESULTS}

The simulation was conducted according to the block diagram shown in Figure 2.

The atomic standard at the TMS and the onboard crystal oscillator can be synchronized to within 1 nanosecond throughout 24 hours, even though the noise of the pseudorange has a 1 nanosecond standard deviation, as shown in Figure 5. The change of range error during simulation is shown in Figure 6. For the orbit information used at the TMS, which corresponds to "Orbit/Delay calculation (with error)" in Figure 2, an initial error of $-5 \mathrm{~m}$ for each axis of ICRF is assumed as measurement error. The difference in range between authentic and measured errors corresponds to the range error. Even though the range error (i.e., orbit estimation) is considerably large $(0-12 \mathrm{~m})$, the proposed method functions correctly.

Using the solutions of $e$ and $k$ of simultaneous equations (3), the time to be adjusted was calculated. The two terms of the time to be adjusted, that is, $e$ and $k / f_{K u}^{2}$, correspond to delays other than ionospheric delay and to the ionospheric delay of the RESSOX control signal using the $K u$-band. As shown in Figure 7, although $e$ and $k / f_{K u}^{2}$ of the time to be adjusted vary by about \pm 30 nanoseconds and 0.5 nanosecond, respectively, because of the noise of the pseudorange, the results of these solutions show good agreement with the actual delays of these origins, that is, the range error

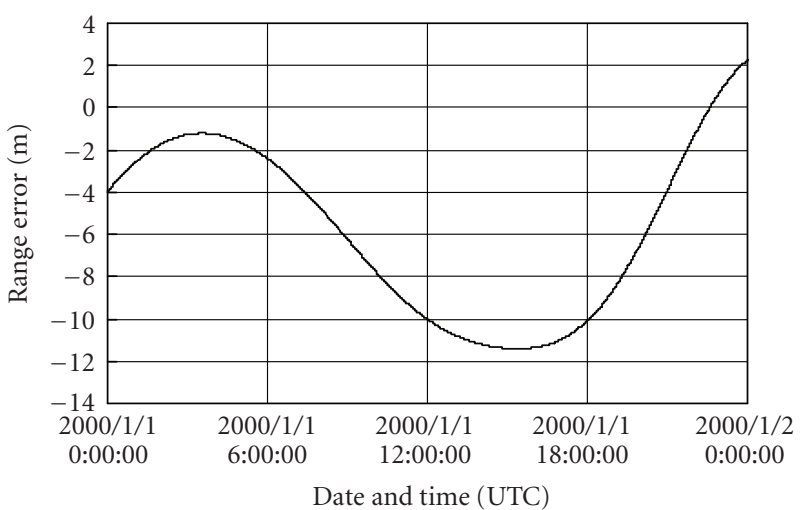

Figure 6: Change of range error during simulation.

plus tropospheric delay and the ionospheric delay shown in Figure 8.

The actual time adjustment command calculated using a combination of 100 elements of the time to be adjusted and the first-order least-squares filter shown in Figure 4 is shown in Figure 9. Since the element of the time to be adjusted, $k / f_{K u}^{2}$, is approximately two orders smaller than that of the time to be adjusted, $e$, the graph shape is similar to that for $e$ in Figure 7. The filter has the effect of reducing the noise.

\section{EFFECT OF ADOPTING THREE FREQUENCIES}

To compare the effects of using three frequencies, synchronization error was evaluated. Three different combinations were investigated: L1 and L2, L1 and L5, and L1, L2, and L5. The combination of L1 and L2 means the current usable combination, and that of L1 and L5 means the most separate frequencies for which a small error is expected. First, we considered the optimum number of data values with the firstorder least-squares filter. The number of data values was increased to 1,000 . The tendency of the number of data values being greater with smaller synchronization error was confirmed, and the best results were obtained in the case of using three frequencies, as shown in Figure 10. In any case, when the number of data values was smaller than 50, the maximum synchronization error was larger than 10 nanoseconds, and the smallest synchronization error was obtained when the number of data values was 1,000. Synchronization using L1/L2/L5 was improved by approximately 15\% compared with that using L1/L2 and by approximately $10 \%$ compared with that using L1/L5.

Next, we compared the effect of the order of the filter, using three frequencies; the results are shown in Figure 11. In the case of the zeroth-order filter, when the number of data values was small, the maximum synchronization error was smaller than 3 nanoseconds; however, it increased when the number of data values was larger than 200. In the case of a first- or higher-order filter, when the number of data values 


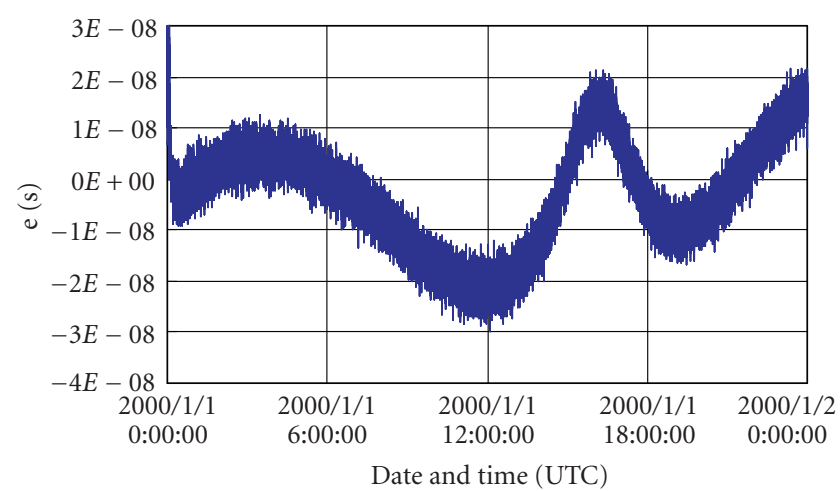

(a)

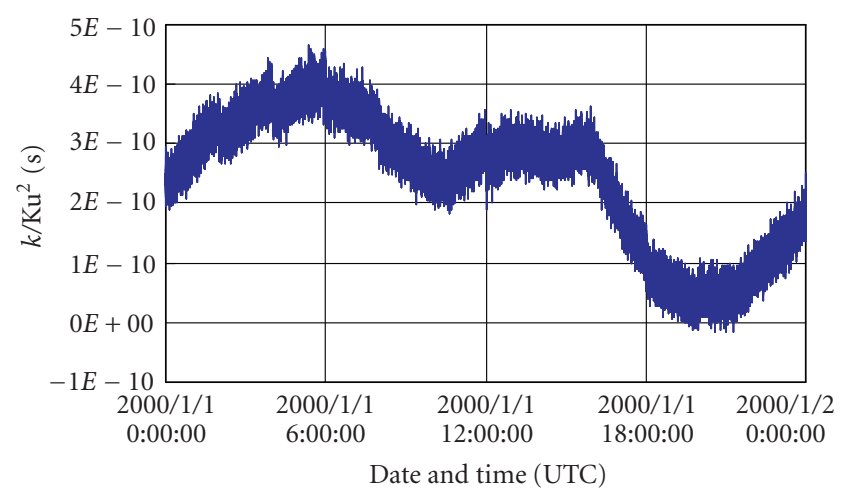

(b)

FIGURE 7: Elements of time to be adjusted. $e$ and $K / f_{K u}^{2}$ correspond to the range error plus tropospheric delay and the ionospheric delay shown in Figure 8, respectively.

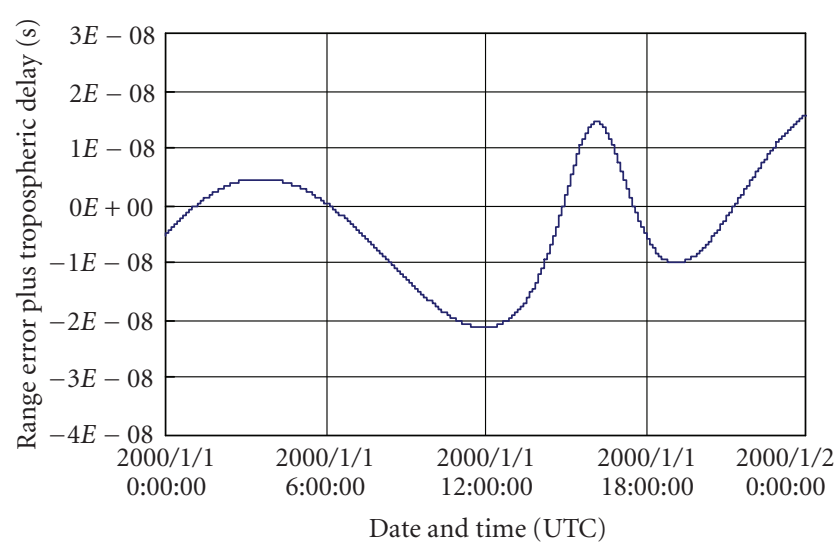

(a)

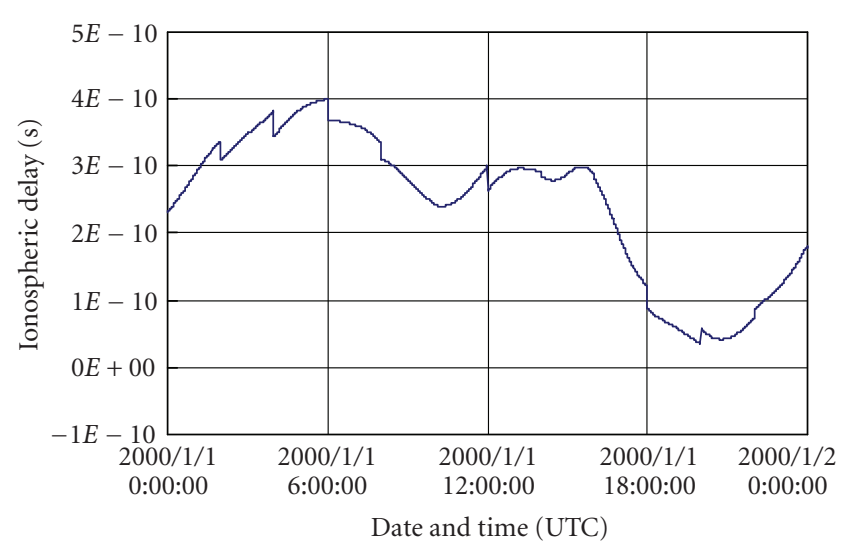

(b)

FIGURE 8: Authentic delay of range error plus tropospheric delay and ionospheric delay.

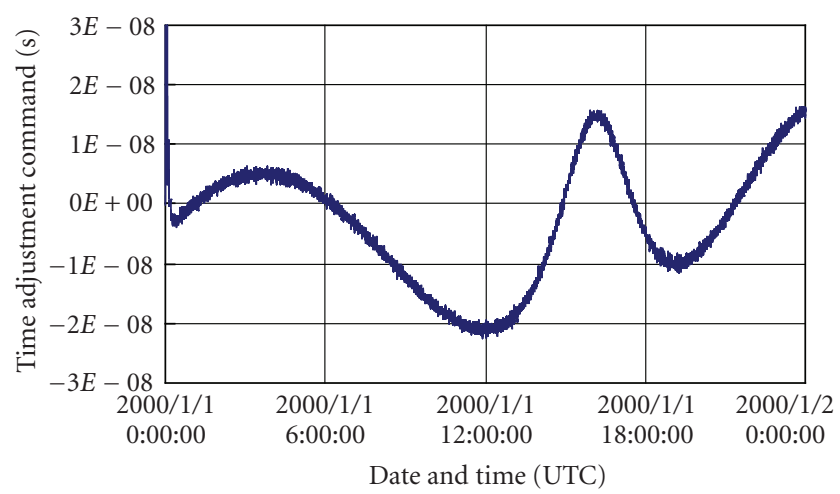

Figure 9: Actual time adjustment command calculated using a combination of 100 elements of time to be adjusted and first-order least-squares filter shown in Figure 4.

was small, the maximum synchronization error became unacceptably large. The smallest maximum synchronization error was obtained when the first-order filter and 1,000 samples were used.

\section{CONCLUSIONS}

This study is summarized as follows.

(1) A new error-adjustment method for remote synchronization of the onboard crystal oscillator (RESSOX) for the QZSS using L1/L2/L5 positioning signals was demonstrated by simulation.

(2) Synchronization to within 1 nanosecond between the onboard crystal oscillator and the ground standard time was achieved in a 24-hour simulation.

(3) The ionospheric delay and the combination of tropospheric delay and range error of the RESSOX control signal were estimated in the calculation and efficiently compensated.

(4) On the ground, the number of data values and the order of the least-squares filter can be changed. The firstorder least-squares filter using 1000 data values and three frequencies is the best, yielding a synchronization error of less than 0.77 nanosecond.

(5) Synchronization using L1/L2/L5 was improved by approximately $15 \%$ compared with that using L1/L2 and by approximately $10 \%$ compared with that using L1/L5. 


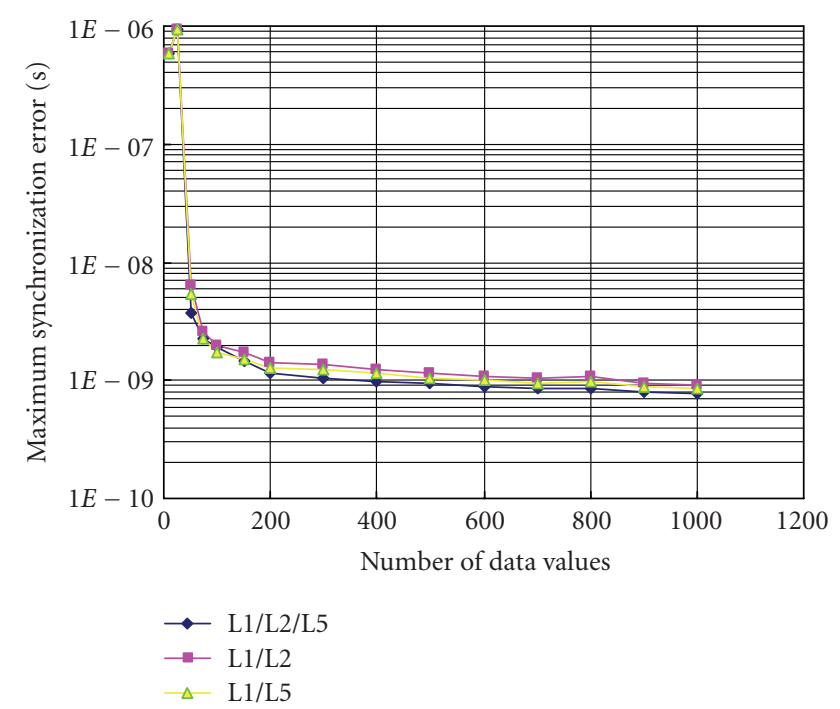

FIgURE 10: Relationship between number of data values and maximum synchronization error. Three different combinations were investigated.

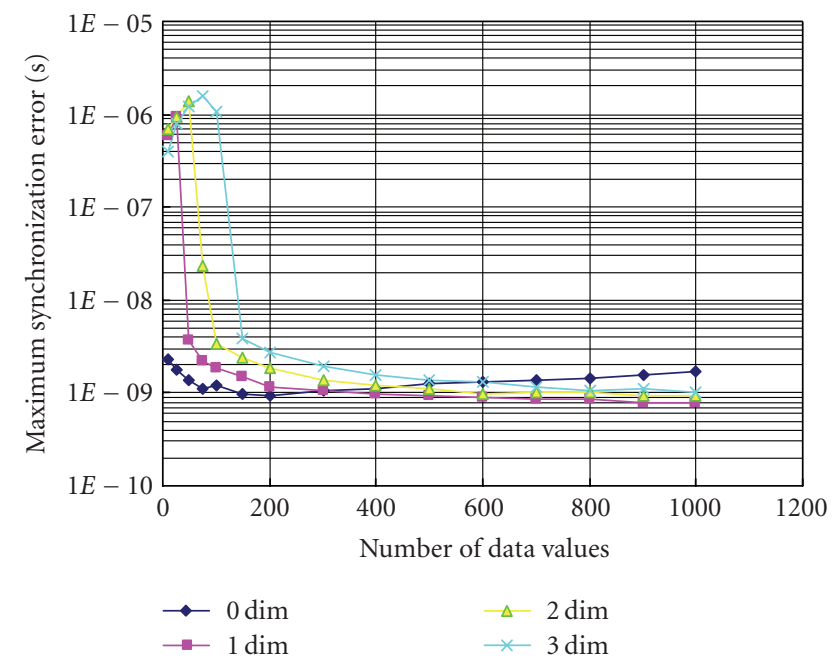

FIgURE 11: Relationship between number of data values and maximum synchronization error. The effect of the order of the filter is compared.

\section{ACKNOWLEDGMENT}

This study was carried out as part of the "Basic Technology Development of Next-Generation Satellites" project promoted by the Ministry of Economics, Trade and Industry (METI) through the Institute for Unmanned Space Experiment Free Flyer (USEF).

\section{REFERENCES}

[1] I. Kawano, M. Mokuno, S. Kogure, and M. Kishimoto, "Japanese experimental GPS augmentation using quasi-zenith satellite system (QZSS)," in Proceedings of the 17th International Technical Meeting of the Satellite Division of the Institute of Navigation (ION-GNSS '04), pp. 175-181, Long Beach, Calif, USA, September 2004.
[2] F. Tappero, T. Iwata, A. Dempster, et al., "Proposal for a novel remote synchronization system for the on-board crystal oscillator of the quasi-zenith satellite system," Navigation, vol. 53, no. 4, pp. 219-229, 2006.

[3] J. J. Suter, L. J. Crawford, B. G. Montgomery, and W. E. Swann, "Syntonics LLC: APL-developed technology makes its commercial debut," Johns Hopkins APL Technical Digest, vol. 22, no. 2, pp. 168-175, 2001.

[4] Japan Aerospace Exploration Agency, Quasi- Zenith Satellite System Navigation Service Interface Specification for QZSS (IS-QZSS) Version 0.0, 2007.

[5] T. Iwata, A. Iwasaki, Y. Fukuyama, et al., "Ground testbed for quasi-zenith satellite remote synchronization system for on-board crystal oscillator," in Proceedings of the 24th Symposium on Space Technology and Science (ISTS '04), Tokyo, Japan, May-June 2004.

[6] T. Iwata, F. Tappero, M. Imae, et al., "Research on remote synchronization system for onboard crystal oscillators of quasizenith satellites," in Proceedings of International Symposium on GPS/GNSS, Sydney, Australia, December 2004.

[7] T. Iwata, F. Tappero, M. Imae, et al., "Ground experiments of remote synchronization system for onboard crystal oscillators of quasi-zenith satellites," in Proceedings of AIAA Guidance, Navigation, and Control Conference, vol. 8, pp. 6250-6264, San Francisco, Calif, USA, August 2005.

[8] T. Iwata, M. Imae, T. Suzuyama, et al., "Ground experiments of remote synchronization for onboard crystal oscillators of quasi-zenith satellites-use of multiple positioning signals for feedback control," in Proceedings of the 38th Annual Precise Time and Time Interval Meeting (PTTI '05), Reston, Va, USA, August 2005.

[9] T. Iwata, F. Tappero, N. Takasaki, et al., "Simulation and ground experiments of remote synchronization system for onboard crystal oscillator of quasi-zenith satellite," Navigation, vol. 53, no. 4, pp. 231-235, 2006.

[10] T. Iwata, Y. Kawasaki, M. Imae, et al., "Remote Synchronization System of Quasi-Zenith Satellites Using Multiple PositioningSignals for Feedback Control," Navigation, vol. 54, no. 2, pp. 99-108, 2007.

[11] User's Manual Stable 32 Version 1.38, Hamilton Technical Service, 2002. 

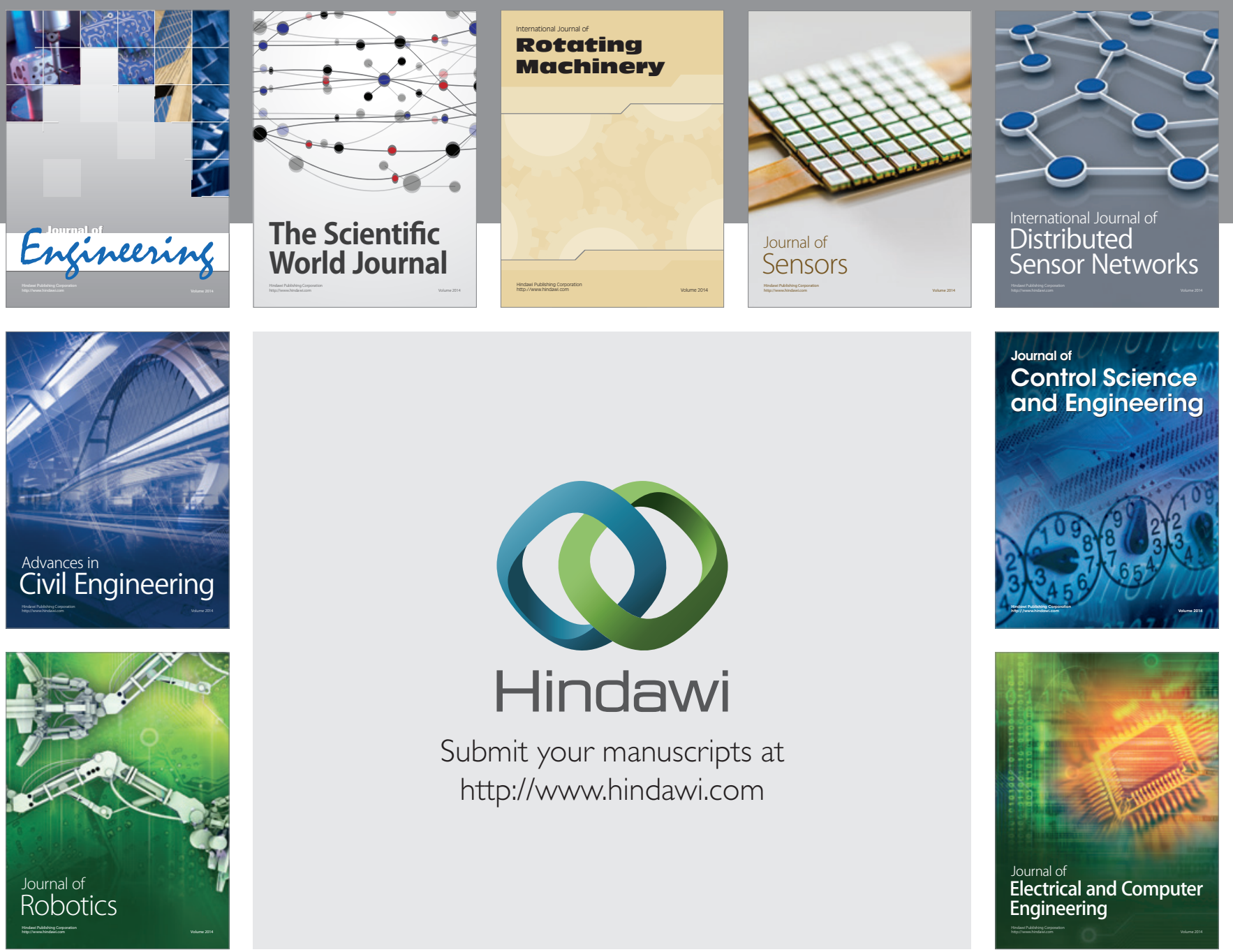

Submit your manuscripts at

http://www.hindawi.com
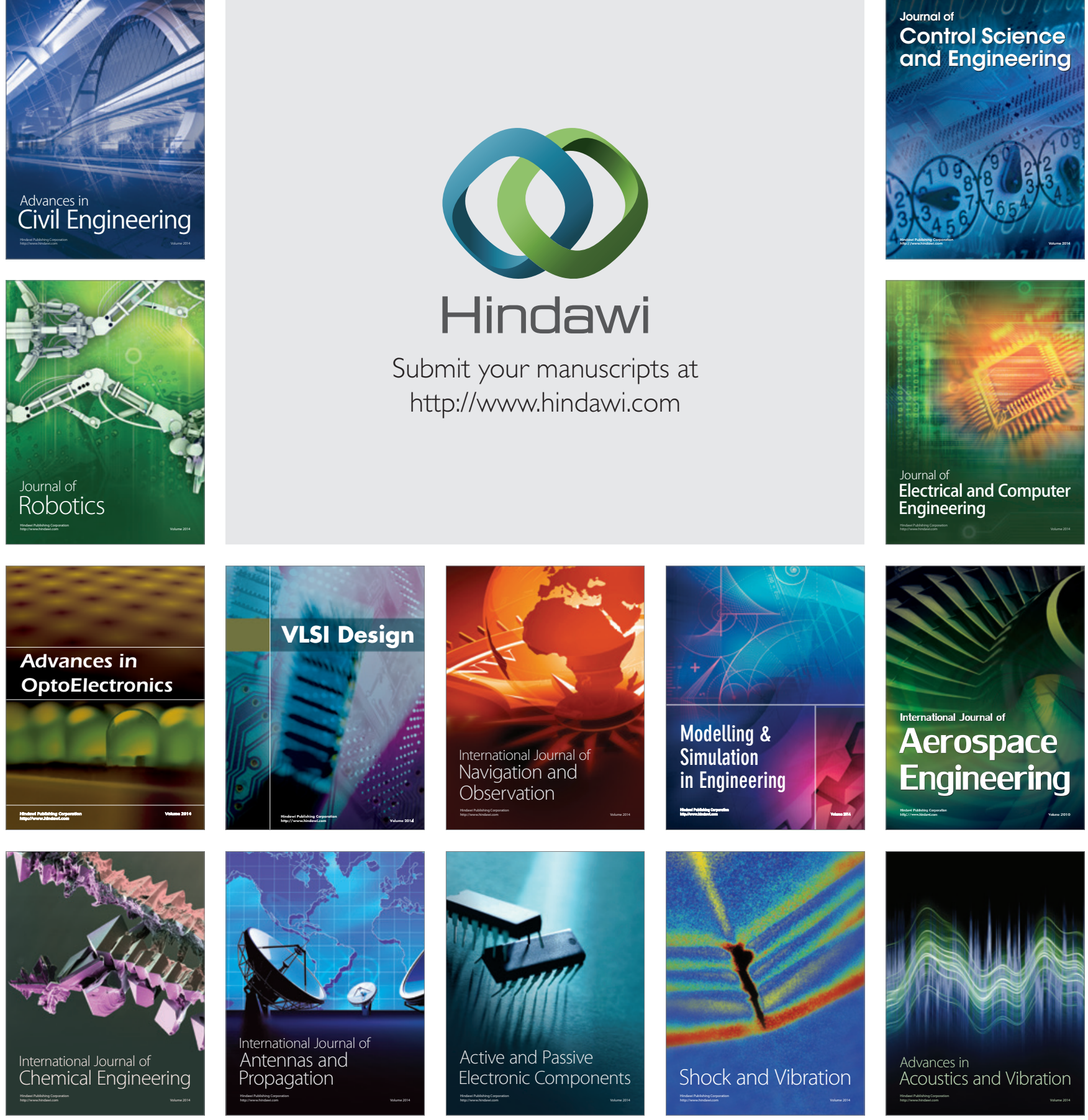